\title{
Prevalence and influencing factors of low back pain among nurses in China: a systematic review and meta-analysis
}

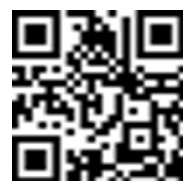

Original article

Dong-Yang Wang ${ }^{\mathrm{a}, *}$, Yu-Yan Sun ${ }^{\mathrm{b}}$

aSchool of Health Science, Mae Fah Luang University, Chiang Rai, Thailand

${ }^{b}$ School of Nursing, Xinxiang Medical University, Xinxiang, Henan 453003, China

Received: 9 June 2020; Accepted: 11 July 2020; Published: 20 December 2020

Abstract: Background: Low back pain (LBP) is one of the most common occupational injuries that nurses experience in their daily work, which ultimately causes disability or difficulty in performing heavy physical activity. There is scarce information that presents a direct figure about the prevalence of LBP in Chinese nurses.

Objective: To systematically review and analyze the prevalence and influencing factors of LBP among nurses in China.

Methods: Related literature extracted from PubMed, Web of Science, Scopus, China National Knowledge Infrastructure databases were selected and reviewed based on the PRISMA guideline. Random effects models were used for data analysis, where heterogeneity was tested through $\mathrm{I}^{2}$. Egger's test was used to analyze the literature publication bias.

Results: The combined data indicated that the prevalence of LBP among nurses in China was $78 \%$ [95\% confidence interval $(\mathrm{Cl})=0.72-0.83]$. The prevalence of LBP among nurses in China has declined in the past decade. Nurses presented a high risk of LBP in terms of female $(\mathrm{OR}=2.38,95 \% \mathrm{Cl}=1.23-3.44, P<0.0001)$, married $(\mathrm{OR}=2.79,95 \% \mathrm{Cl}=1.57-3.86, P<0.0001)$, $24 \leq \mathrm{BMI}<28(\mathrm{OR}=2.03,95 \% \mathrm{Cl}=1.18-3.08, P<0.0001)$, and working for more than ten $>10$ years $(\mathrm{OR}=2.54,95 \%$ $\mathrm{Cl}=2.03-3.32, P<0.0001)$.

Conclusions: There is a high prevalence of LBP among nurses in China. Interventions that include healthy dietary habits and physical activity mainly focusing on female married nurses who are working for at least 10 years should be implemented. Furthermore, nurses ought to apply ergonomic principles for personal protection at the workplace.

Keywords: low back pain $\bullet$ nurse $\bullet$ meta-analysis $\bullet$ China $\bullet$ chronic disease

(c) Shanxi Medical Periodical Press.

\section{Introduction}

Low back pain (LBP) is defined as pain, discomfort, and functional limitation in the area from T12 rib below to hip fold above, excluding primary structural pathological changes such as disc herniation and spinal stenosis. ${ }^{1,2}$ It mainly involves back pain, radicular pain, and involvement pain. ${ }^{3}$ At present, LBP is one of the

How to cite this article: Wang DY, Sun YY. Prevalence and influencing factors of low back pain among nurses in China: a systematic review and meta-analysis. Front Nurs. 2020;4:329-336. 
most severe burdens of noncommunicable diseases in the world. It accounts for disability among 60.1 million people worldwide. ${ }^{4}$ Besides, up to $\$ 87.6$ billion in medical spending for LBP has also brought enormous economic pressure on governments and people, especially in the middle- and low-income countries. ${ }^{5}$

The worldwide prevalence of LBP in adults was about $40 \%$, while the incidence is around $38 \%$ each year. ${ }^{6}$ Owing to occupational physical and emotional factors, the risk of LBP in healthcare workers is much higher than that of construction workers and other manufacturing and construction practitioners. ${ }^{7}$ Health workers are exposing to those LBP risk factors due to the involvement in repeated treatment and rehabilitation activities for patients, which includes heavy manual handling, relocation of patients, and working in extremely awkward positions. ${ }^{8}$

A study in Bangladesh is evident that nurses are more vulnerable to LBP among occupational populations in the healthcare services sector. ${ }^{9}$ It has been reported that the global prevalence of LBP among nurses around the world was $40-90 \%$ and developing countries with a shortage of health workforce are at higher risk. ${ }^{10}$ Turkish and Slovenian studies found the prevalence of LBP among nurses at $84.2 \%$ and $85.9 \%$, respectively. ${ }^{11,12}$ Guan et al. stated the prevalence of LBP in Chinese nurses at $51.63 \% .^{13}$

Several studies have indicated that the prevalence and factors related to LBP among nurses are from different geographical locations in China. However, the comprehensive nationwide data about the prevalence rate of LBP and its influencing factors in Chinese nurses are limited. Similarly, to facilitate valid and productive efforts to interpret and replicate the prevalence and influencing factors of LBP, an understanding of the current state of the literature is necessary. Therefore, this article aimed to assess the prevalence and influencing factors of LBP among nurses in China.

\section{Methods}

\subsection{Data sources and searches}

The systematic review and meta-analysis of this study were performed based on the PRISMA guidelines. The literature was searched from January to February 2019 using the following databases: PubMed, Web of Science, Scopus, and China National Knowledge Infrastructure (CNKI). Original articles published from 2010 to 2019 were identified by using the keywords "low back pain," "nurse," "prevalence," "incidence," and "current status." In addition, keywords with the medical subject headings (MeSh) using a conjunctions word, such as AND, NOT, OR, were used in the "Advanced Searches" of the international database. Furthermore, researchers paid attention to conference papers included in related databases. The citations had been entered in the reference management software Mendeley, Version 1.19 (Elsevier, London, UK).

\subsection{Study selection}

Two authors searched the database based on titles and abstracts to exclude duplicate and unrelated literature. In contrast, the other authors reviewed the full text of literature based on the results of the first screening independently. If there were any discrepancies, the first author performed as the tiebreaker.

The literature selection process followed the literature's inclusion and exclusion criteria of this study. The cross-sectional study that reported the prevalence and influencing factors of LBP among more than 50 nurses in mainland China was the inclusion criteria. However, the literature that includes study population other than in-service nurses, incomplete study results or errors, and languages other than English or Chinese was excluded.

\subsection{Data extraction and quality assessments}

Two independent researchers performed data extraction. Researchers encoded related variables in the literature based on variable characteristics. Extracted variables were author name, year of publication, study site, study population, sample size, type of standard questionnaire, gender, age, marital status, education background, $\mathrm{BMI}$, year of working experience, and prevalence of LBP. Furthermore, the adjusted odds ratios (AOR) with confidence intervals (Cls) of variables, that is, gender, age, BMI, educational background, and work experience variables were also extracted.

The researchers conducted a preliminary evaluation of the selected literature following the Australian Joanna Briggs Institute (JBI) Reviewers Manual. ${ }^{14}$ The scope of the evaluation included the evaluation research framework, sampling techniques, sample size, research settings, statistical analysis, inclusion and exclusion criteria, and so on. The literature with a score higher than $70 \%$ evaluated as low-risk literature.

\subsection{Data synthesis and analysis}

This study used Stata software, Version 16.0 (StataCorp, College Station, Texas, USA) for meta-analysis. The researchers first collected the prevalence from all studies, then calculated the variance of LBP based on the binomial distribution, and assigned the weight of each study based on the inverse of the variance. The 
$\mathrm{I}^{2}$ statistic was used to assess heterogeneity between studies and categorized into four parts based on the $1^{2}$ score: no heterogeneity (0\%), low (25-50\%), moderate (50-75\%), and high (>75\%). ${ }^{15}$

The random effects model was used to calculate the combined prevalence of LBP and the log-transformed odds ratio. Researchers examined the causes of heterogeneity in the study by subgroup analysis, and the Egger's test was performed to analyze the literature publication bias. The $p<0.05$ was considered statistically significant. Data about the prevalence of LBP among nurses were presented through a forest chart. The line chart used to indicate the trend of LBP among nurses from 2010 to 2019 .

\section{Results}

\subsection{The result of the literature search}

Through database keywords search, a total of 514 research papers were identified. A total of 14 literature were selected for meta-analysis after excluding duplicate literature, literature with unrelated title and abstract, irrelevant in-text information, literature that not met eligibility criteria, and high-risk literature, as shown in Figure 1. The total number of participants involved in those studies was 8,179 , and the quality scores of each selected literature exceeded $80 \%$ (Table 1).

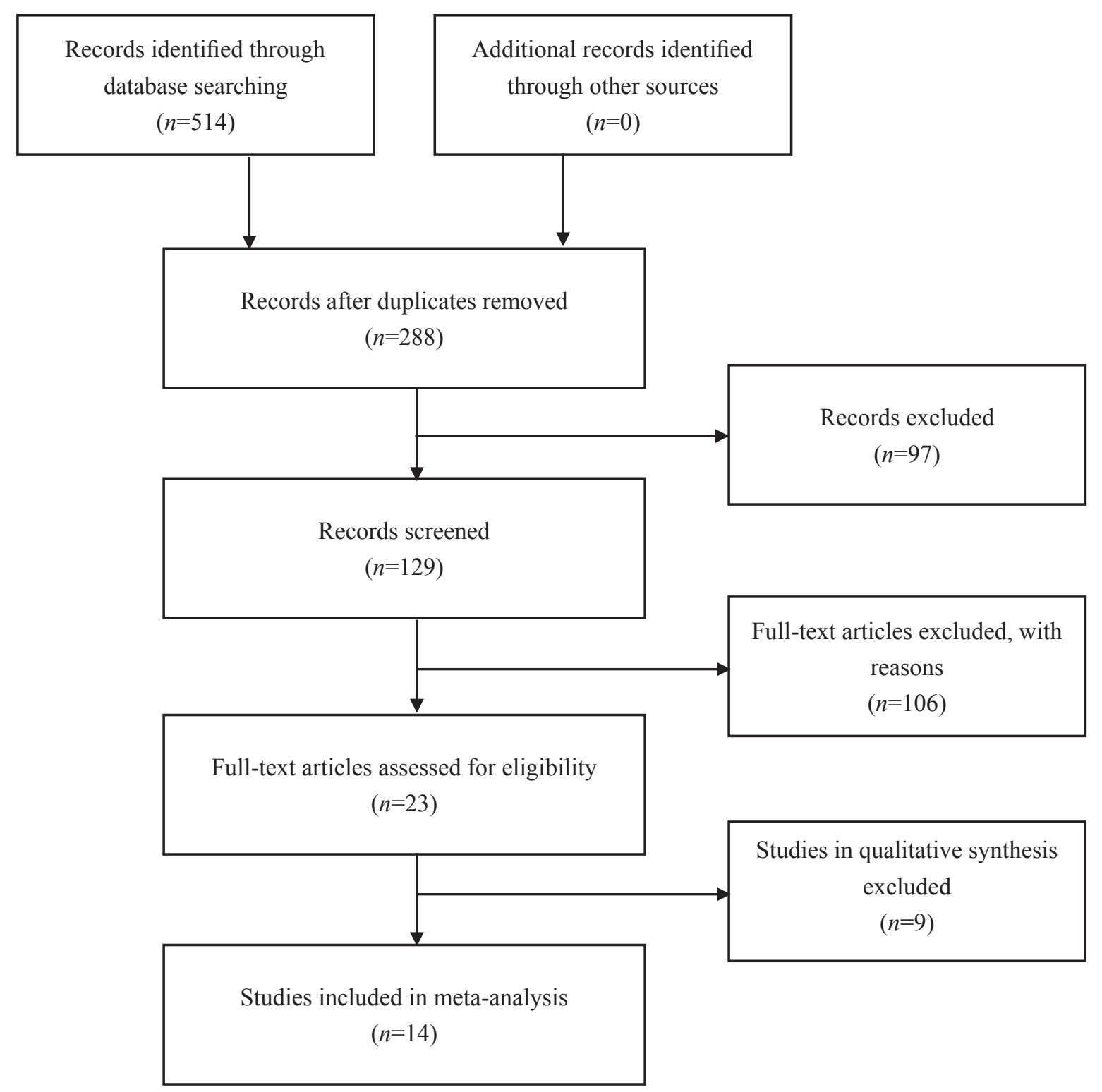

Figure 1. PRISMA process of the systematic literature search. 


\begin{tabular}{|c|c|c|c|c|c|c|c|}
\hline Author & Year & Study sites & Sample sizes & Gender & Age (years) & Measures & Literature quality (\%) \\
\hline Chen et al. ${ }^{16}$ & 2010 & Quanzhou & 171 & $100 \%$ female & $20-46$ & Self-made questionnaire & 90 \\
\hline Deng et al. ${ }^{17}$ & 2010 & Dongguan & 120 & $100 \%$ female & $17-56$ & Self-made questionnaire & 80 \\
\hline Xu et al. ${ }^{18}$ & 2010 & Harbin & 108 & $88.0 \%$ female & $19-49$ & Self-made questionnaire & 100 \\
\hline Yin et al..$^{19}$ & 2013 & Hebei & 672 & $89.3 \%$ female & $N / A$ & Self-made questionnaire & 80 \\
\hline Wei et al. ${ }^{20}$ & 2014 & Nanjing & 256 & $91.0 \%$ female & $22-40+$ & Self-made questionnaire & 100 \\
\hline Wang et al. ${ }^{21}$ & 2016 & Yinchuan & 909 & $\mathrm{~N} / \mathrm{A}$ & $31.93 \pm 7.42$ & Self-made questionnaire & 90 \\
\hline Zhang et al. ${ }^{22}$ & 2017 & Shizuishan & 172 & $100 \%$ female & $20-35+$ & Self-made questionnaire and NRS scale & 100 \\
\hline Zhang et al. ${ }^{23}$ & 2017 & Lvliang & 100 & $93 \%$ female & $\mathrm{N} / \mathrm{A}$ & NRS scale and BDI scale & 90 \\
\hline Tang et al. ${ }^{24}$ & 2018 & Mainland China & 1279 & $93.7 \%$ female & $20-45$ & VAS scale and $F A B Q$ questionnaire & 100 \\
\hline Guan et al. ${ }^{13}$ & 2019 & Mainland China & 2458 & $96.4 \%$ female & $20-30+$ & Self-made questionnaire and VAS scale & 100 \\
\hline Huang ${ }^{25}$ & 2019 & Zunyi & 203 & $\mathrm{~N} / \mathrm{A}$ & $20-50$ & Self-made questionnaire & 90 \\
\hline Li et al. ${ }^{26}$ & 2019 & Sichuan & 783 & $98.5 \%$ female & $19-55$ & RMDQ and $F A B Q$ questionnaires & 100 \\
\hline$W u^{27}$ & 2019 & Nanyang & 648 & $N / A$ & $20-53$ & Self-made questionnaire and VAS scale & 80 \\
\hline Zhang et al. ${ }^{28}$ & 2019 & Shandong & 498 & $85.3 \%$ female & $31.1 \pm 7.6$ & Self-administered questionnaire & 100 \\
\hline
\end{tabular}

Table 1. Characteristics of literature included in a systematic review.

\subsection{The prevalence and trend of LBP among nurses}

According to statistics included in 14 literature, the lowest and highest prevalence rates of LBP were $63.0 \%$ and $92.4 \%$, respectively. The meta-analysis using a random effects model showed that the prevalence of LBP among Chinese nurses was 78\% [95\% confidence interval $(\mathrm{Cl})=0.72-0.83$, as shown in Figure 2. From the study findings, the researchers found the gradual decrement in the prevalence of LBP among Chinese nurses over the last decade. However, the prevalence of LBP was still seemed to be high (Figure 3 ).

\subsection{Subgroup analysis}

According to the results of the subgroup analysis, female nurses $(75.6 \%, 95 \% \mathrm{Cl}=67.4-83.8, p<0.001)$ had higher prevalence of LBP than male $(46.4 \%, 95 \%$ $\mathrm{Cl}=27.9-54.8, p<0.001)$. 35-55 years old nurses (75.5\%, 95\% Cl $=70.9-80.1, p<0.0001)$ and married nurses $(69.8 \%, 95 \% \mathrm{Cl}=49.9-89.6, p<0.0001) \mathrm{had}$ proportion of LBP compared with 19-34 years old nurses (64.9\%, 95\% Cl $=53.0-76.7, p<0.0001)$ and unmarried nurses $(33.2 \%, 95 \% \mathrm{Cl}=16.8-49.7, p<0.0001)$, respectively. Among nurses with a bachelor's degree or above, the proportion of LBP was $63.5 \%(95 \% \mathrm{Cl}=48.9$ $71.5, p=0.001$ ) which was higher in comparison with nurses with junior college-level education (69.3\%) and secondary school level education (73.6\%). Regarding $\mathrm{BMI}$ of the nurses, obese or overweight nurses $(75.6 \%$, $95 \% \mathrm{Cl}=71.7-79.5, p=0.006)$ reported having high LBP than normal BMI (50.1\%) and low BMI nurses
$(37.7 \%)$. Furthermore, nurses who had worked for $>10$ years $(82.2 \%, 95 \% \mathrm{Cl}=76.8-87.6, p=0.014)$ had a higher prevalence than nurses who were working for $5-10$ years $(72.5 \%)$ and $<5$ years $(56.3 \%)$. The details are given in Table 2 .

\subsection{The risk factors of LBP}

Female nurses were 2.38 times more likely to have LBP than male nurses $(95 \% \mathrm{Cl}=1.23-3.44, p<0.0001)$. Married nurses were 2.79 times more likely to have LBP than unmarried nurses $(95 \% \mathrm{Cl}=1.57-3.86, p<0.0001)$. Similarly, obese or overweight nurses $(\mathrm{OR}=2.03$, $95 \% \mathrm{Cl}=1.18-3.08, p<0.0001$ ) have a higher risk of getting LBP than nurses with normal BMI. Nurses who were working for $>10$ years had 2.54 times $(\mathrm{OR}=2.54$, $95 \% \mathrm{Cl}=2.03-3.32, p<0.0001)$ more chance to experience LBP than nurses who were working for $<5$ years.

\section{Discussion}

From the findings of this literature review, researchers found that the prevalence of LBP among Chinese nurses was $78 \%$, which shows that the current government measures are not effective enough in preventing and controlling LBP among Chinese nurses. Similar to the current result, studies from Turkey, Slovenia, and Saudi Arabia reported the prevalence of LBP $>60 \%$, which indicates that developing countries have a high risk of LBP among nurses. ${ }^{12,29,30}$ However, the prevalence rate of LBP in nurses of developed countries, such as Japan (11.8\%) and Sweden 


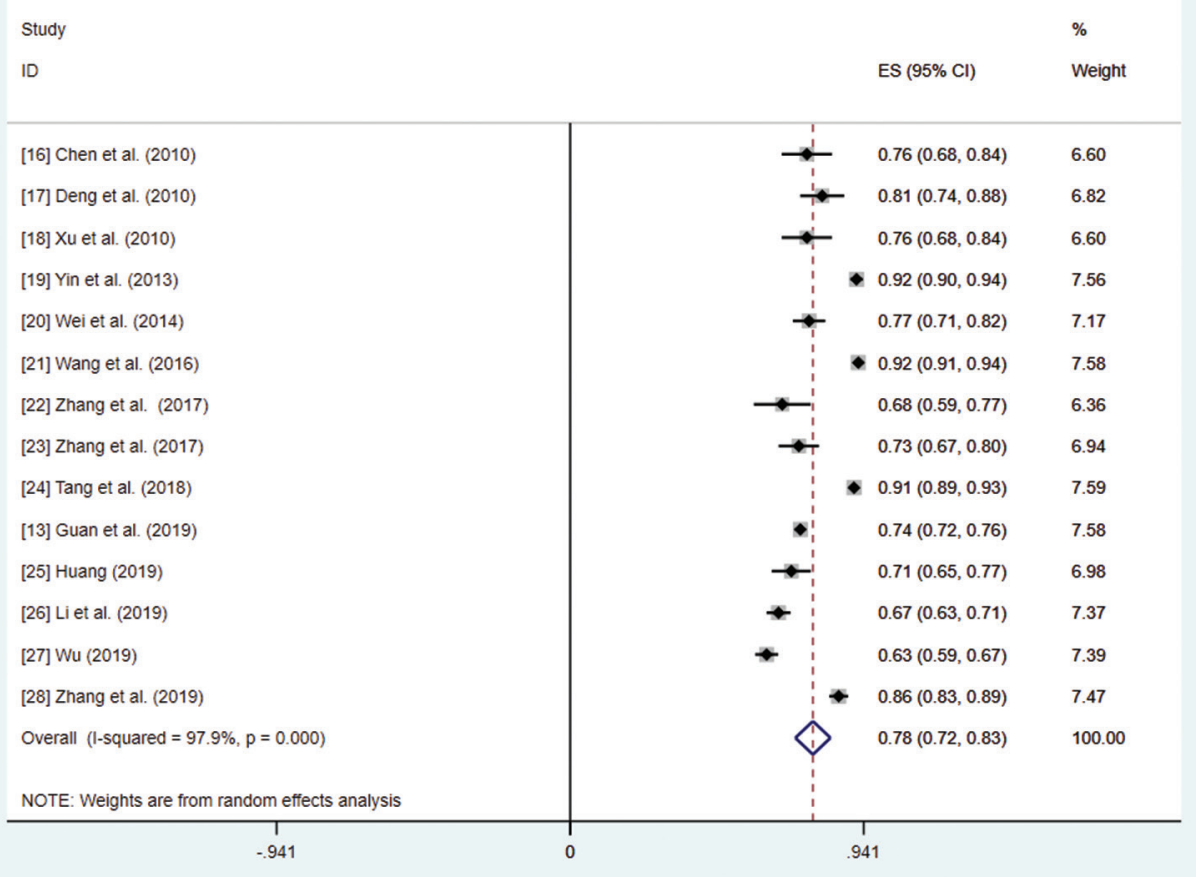

Figure 2. The prevalence of low back pain.

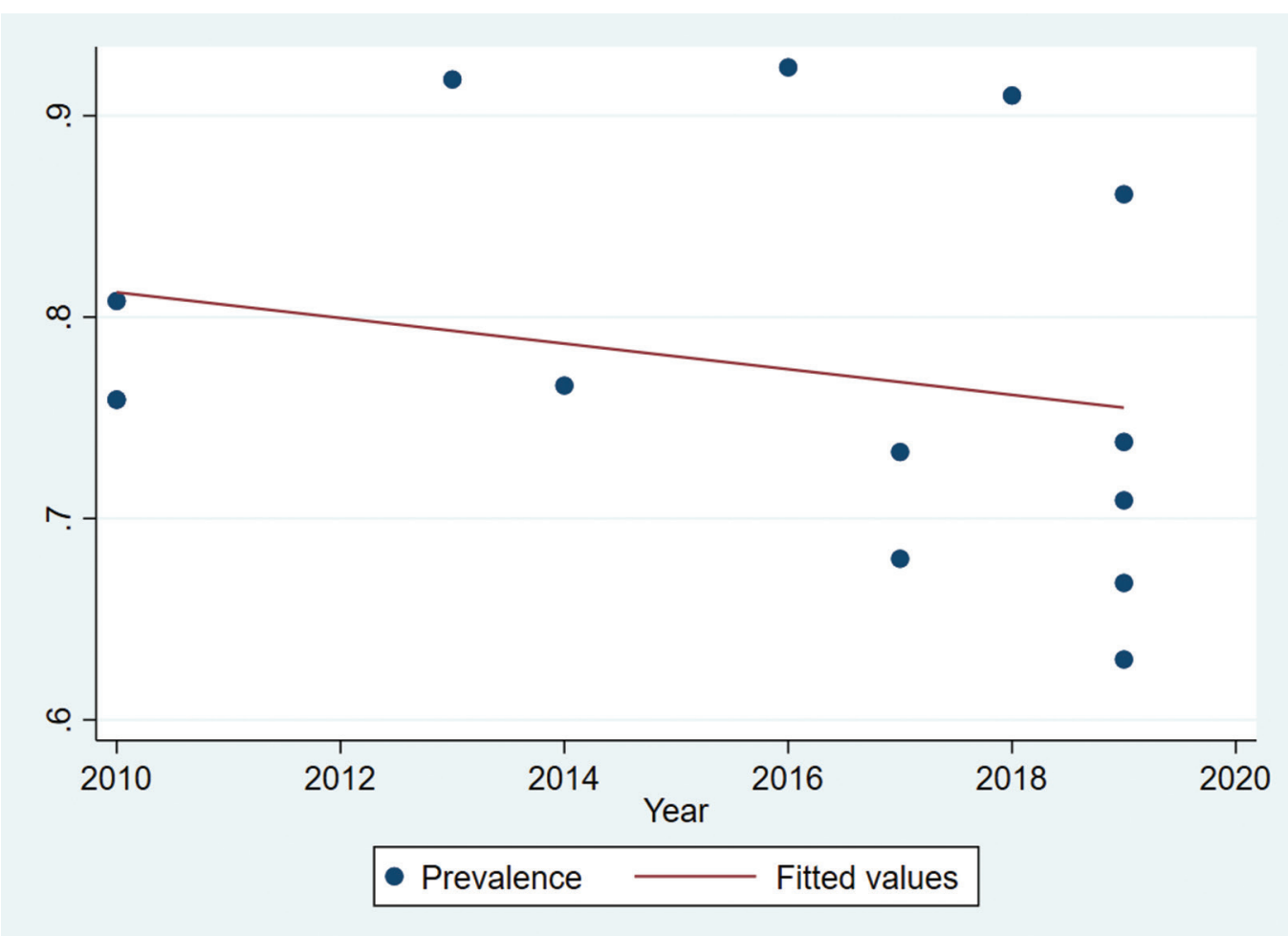

Figure 3. The trend of low back pain from 2010 to 2019. 


\begin{tabular}{|c|c|c|c|c|}
\hline \multirow[t]{2}{*}{ Variables } & \multirow[t]{2}{*}{ Prevalence (\%) } & \multicolumn{2}{|c|}{$\mathrm{Cl}(\%)$} & \multirow[t]{2}{*}{$P$-value } \\
\hline & & Lower & Upper & \\
\hline \multicolumn{5}{|l|}{ Gender } \\
\hline Male & 46.4 & 27.9 & 54.8 & 0.001 \\
\hline Female & 75.6 & 67.4 & 83.8 & 0.001 \\
\hline \multicolumn{5}{|l|}{ Age (years) } \\
\hline $19-34$ & 64.9 & 53.0 & 76.7 & 0.0001 \\
\hline $35-55$ & 75.5 & 70.9 & 80.1 & 0.0001 \\
\hline \multicolumn{5}{|l|}{ Marital status } \\
\hline Unmarried & 33.2 & 16.8 & 49.7 & 0.0001 \\
\hline Married & 69.8 & 49.9 & 89.6 & 0.0001 \\
\hline \multicolumn{5}{|l|}{ Education background } \\
\hline Secondary school & 73.6 & 67.8 & 79.3 & 0.0001 \\
\hline Junior college & 69.3 & 56.9 & 76.6 & 0.003 \\
\hline $\begin{array}{l}\text { Bachelor's degree or } \\
\text { above }\end{array}$ & 63.5 & 48.9 & 71.5 & 0.001 \\
\hline \multicolumn{5}{|l|}{$B M I$} \\
\hline $24 \leq \mathrm{BMI}<28$ & 75.6 & 71.7 & 79.5 & 0.006 \\
\hline $18.5 \leq \mathrm{BMI}<24$ & 50.1 & 46.4 & 53.8 & 0.0001 \\
\hline $\mathrm{BMI}<18.5$ & 37.7 & 30.1 & 45.2 & 0.001 \\
\hline \multicolumn{5}{|c|}{ Years of working experience } \\
\hline$<5$ & 56.3 & 43.5 & 69.0 & 0.0001 \\
\hline $5-10$ & 72.5 & 64.1 & 80.9 & 0.0001 \\
\hline$>10$ & 82.2 & 76.8 & 87.6 & 0.014 \\
\hline
\end{tabular}

Note: $\mathrm{Cl}$, confidence interval; LBP, low back pain.

Table 2. The demographic information of LBP.

(19.0\%), found to be lower compared with the current study result. ${ }^{31,32}$ The probable reason behind the high prevalence of LBP in nurses of developing countries, including China, could be due to the inadequate health workforce, unmatched nurse-patient ratio, and working in the unhealthy work settings with high workload. ${ }^{33}$

The trend analysis of LBP among Chinese nurses found a downward trend of LBP in the past 10 years. Nonetheless, the overall declination was seemed to be slow. It reflects that some hospitals in China have begun to address the problem of LBP among nurses by adopting LBP prevention strategies, which appeared as less fruitful. Similar reasons were illustrated in the study from Guan et al. ${ }^{13}$

This article indicated a significant association between LBP and female nurses. The previous study supported the current result that female nurses are at higher risk of LBP, which could be exacerbated by the menstrual cramps and their pain threshold. ${ }^{34}$ Marital status was found as another influencing factor of LBP in nurses. The relevant Chinese literature has shown that married female nurses involve in both domesticwork and occupational-work, which lodged them to the high susceptibility of LBP. ${ }^{18} \mathrm{~A}$ study by Danish added up the evidence that married nurses are more prone to LBP during their pregnancy. Pregnancy causes natural softening and stretching of the ligaments, which puts a strain on the joints of the pelvis and lower back, resulting in LBP. ${ }^{35}$

The results of this study identified that nurses working for $>10$ years were 2.54 times more likely to develop LBP than nurses working for $<5$ years. The relevant research shows that long-term repeated bending postures of clinical nurses may increase uneven spinal pressure causes LBP. ${ }^{16} \mathrm{~A}$ study mentioned about the professionalism of nursing work requires continuous ability to grow their skill and adopt in the changing measures as well as health technologies. The majority of the senior nurses are not able to match the changing demand skill and cannot apply the mechanical principles correctly in various operations leads to the deterioration of bones, muscles, and other physiological functions possessing the high LBP risk. ${ }^{24}$

In this article, obese or overweight nurses found to have a higher risk of getting LBP than nurses with normal BMI, which is similar to the result reported in the study of Zhang et al.. ${ }^{28}$ Several studies identified that people with increased BMI and unhealthy lifestyles, including lack of physical activity and inappropriate diet, were more likely to suffer from chronic LBP. ${ }^{36,37}$ A study by $\mathrm{Li}$ et al. demonstrated that due to a high workload and less leisure time, nurses are practicing unhealthy dietary habits and inadequate physical activity, which are known significant drivers of increased BMI and LBP itself. ${ }^{26}$

The nationwide survey is needed to uncover the significant stressors of LBP among nurses, which could provide the platform for the development of effective policy, plans, and interventions to prevent LBP and its complications among Chinese nurses.

\section{Conclusions}

The prevalence of LBP among Chinese nurses in this study was found to be decreased in the past decade. However, the prevalence rate still seemed to be higher compared with developed countries. The interventions that include healthy dietary habits and regular physical activity should be implemented to control the burden of LBP, focusing on female married nurses who are working for at least 10 years. The government health departments and policy-makers need to update the current LBP prevention guidelines and implement them 
accordingly to overcome the foreseeable risk of LBP and its complications.

\section{Limitations}

This article has a high degree of heterogeneity, mainly caused by different factors such as geographical, hospital grades, departments, and follow-up time of each study, which limits the scope of interpretation of this article.

\section{References}

1. Jiménez-Ávila JM, Rubio-Flores EN, GonzálezCisneros AC, Guzmán-Pantoja JE, GutiérrezRomán EA. Directrices en la aplicación de la guía de práctica clínica en la lumbalgia [Guidelines on the application of the clinical practice guideline on low back pain]. Cirugia y cirujanos. 2018;86:29-37.

2. Norton G, McDonough CM, Cabral HJ, Shwartz M, Burgess JF. Classification of patients with incident non-specific low back pain: implications for research. Spine J. 2016;16:567-576.

3. Urits I, Burshtein A, Sharma M, et al. Low back pain, a comprehensive review: pathophysiology, diagnosis, and treatment. Curr Pain Headache Rep. 2019;23:23.

4. Sharma S, Traeger AC, Mishra SR, Sharma S, Maher CG. Delivering the right care to people with low back pain in low- and middle-income countries: the case of Nepal. J Global Health. 2019;9:010304.

5. TraegerA, BuchbinderR, HarrisI, MaherC.Diagnosis and management of low-back pain in primary care. Can Med Assoc J. 2017;189:E1386-E1395.

6. Manchikanti L, Singh V, Falco FJ, Benyamin RM, Hirsch JA. Epidemiology of low back pain in adults. Neuromodulation. 2014;17:3-10.

7. Landry MD, Raman SR, Sulway C, Golightly YM, Hamdan E. Prevalence and risk factors associated with low back pain among health care providers in a Kuwait hospital. Spine. 2008;33:539-545.

8. Awosan KJ, Yikawe SS, Oche OM, Oboirien M. Prevalence, perception and correlates of low back pain among healthcare workers in tertiary health institutions in Sokoto, Nigeria. Ghana Med J. 2017;51:164-174.

9. Sanjoy SS, Ahsan GU, Nabi H, Joy ZF, Hossain A. Occupational factors and low back pain: a crosssectional study of Bangladeshi female nurses. BMC Res Notes. 2017;10:173.

10. Attar SM. Frequency and risk factors of musculoskeletal pain in nurses at a tertiary centre

\section{Ethics approval}

This article was approved by the ethics committee of Mae Fah Luang University (IRB approval number: 129/2562).

\section{Conflicts of interest}

All contributing authors declare no conflicts of interest.

in Jeddah, Saudi Arabia: a cross sectional study. BMC Res Notes. 2014;7:61.

11. Ovayolu O, Ovayolu N, Genc M, Col-Araz N. Frequency and severity of low back pain in nurses working in intensive care units and influential factors. Pak J Med Sci. 2014;30:70-76.

12. Skela-Savič B, Pesjak K, Hvalič-Touzery S. Low back pain among nurses in Slovenian hospitals: crosssectional study. Int Nurs Rev. 2017;64:544-551.

13. Guan J, Wu DM, Xie XP, et al. Occupational factors causing pain among nurses in Mainland China. Med Sci Monit. 2019;25:1071-1077.

14. Zeng XT, Zhang YG, Kwong JSW, et al. The methodological quality assessment tools for preclinical and clinical studies, systematic review and meta-analysis, and clinical practice guideline: a systematic review. J Evidence-Based Med. 2015;8:2-10.

15. Ades AE, Lu G, Higgins JP. The interpretation of random-effects meta-analysis in decision models. Med Decis Making. 2015;25:646-654.

16. Chen $Q Q$, Huang LP, Lin XL, Ye BR. Research of the conditions of low back pain and its correlating factors among nurses in some 3A-Level hospitals in Quanzhou City. J Nurs Admin. 2010;10:490-492 (in Chinese).

17. Deng WD, Wei N. Cause analysis and preventing method of nurses prone to lumbar disc herniation (LIDP). China Med Herald. 2010;7:160-161 (in Chinese).

18. $\mathrm{Xu} Y$, Ren $\mathrm{YN}$. Survey on occupational factors of low back pain for clinical nurses in a hospital. J Nurs. 2010;17:23-26 (in Chinese).

19. Yin LS, Zhang YX, Li XJ, et al. Investigation and research of nurses in 12 class III Grade I hospitals ICU of low back pain. Nurs Pract Res. 2013;10:6-7 (in Chinese).

20. Wei JC, Yang ML. Low back pain of nurses in operating rooms and its influencing factors. J Nurs Sci. 2014;29:45-47 (in Chinese). 
21. Wang XL, Ren JQ, Liu J. The status and influencing factors of low back pain of 909 nurses in three tertiary grade A hospitals. Chin Nurs Manage. 2016;16:61-64 (in Chinese).

22. Zhang L, Quan Y, Zhao M. Investigation of low back pain in clinical nurses and analysis of influencing factors. Chin Gen Pract Nurs. 2017;15:2311-2313 (in Chinese).

23. Zhang XM, Zhang ZH, Zhu XZ, et al. Occupational related factors of low back pain in hospital nurses. Ningxia Med J. 2017;39:671-672 (in Chinese).

24. Tang LM, Ning WJ, Sun HL, Zhang Z, Tian SZ. Multi-center investigation and the relative factors of the clinical nurse occupational low back pain. Chin J Mod Nurs. 2018;24:3292-3295 (in Chinese).

25. Huang WM. Investigation and protection of low back pain and occupation-related factors in nurses of a third-level general hospital in Zunyi. J Qiqihar Univ Med. 2019;40:1011-1013 (in Chinese).

26. Li L, Deng $X$, Zhang $\mathrm{H}$, et al. A cross-sectional survey of low back pain in nurses working in orthopedic departments. Workplace Health Saf. 2019;67:218-230.

27. Wu XJ. Occupational risk factors of low back pain in nurses. China Occup Med. 2019;46:194-197 (in Chinese).

28. Zhang $\mathrm{Q}$, Dong $\mathrm{H}$, Zhu $\mathrm{C}$, Liu $\mathrm{G}$. Low back pain in emergency ambulance workers in tertiary hospitals in China and its risk factors among ambulance nurses: a cross-sectional study. BMJ Open. 2019;9:e029264.

29. Çınar-Medeni Ö, Elbasan B, Duzgun I. Low back pain prevalence in healthcare professionals and identification of factors affecting low back pain. $J$ Back Musculoskelet Rehabil. 2017;30:451-459.
30. Gaowgzeh R. Low back pain among nursing professionals in Jeddah, Saudi Arabia: Prevalence and risk factors. J Back Musculoskelet Rehabil. 2019;32:555-560.

31. Yoshimoto T, Oka H, Fujii T, et al. Survey on chronic disabling low back pain among care workers at nursing care facilities: a multicenter collaborative cross-sectional study. J Pain Res. 2019;12:1025-1032.

32. Dhaini SR, Zúñiga $F$, Ausserhofer $D$, et al. Care workers health in Swiss nursing homes and its association with psychosocial work environment: a crosssectional study. Int J Nurs Stud. 2016;53:105-115.

33. Shieh SH, Sung FC, Su CH, Tsai Y, Hsieh VC. Increased low back pain risk in nurses with high workload for patient care: a questionnaire survey. Taiwan J Obstet Gynecol. 2016;55:525-529.

34. Taulaniemi A, Kankaanpää M, Tokola K, Parkkari J, Suni JH. Neuromuscular exercise reduces low back pain intensity and improves physical functioning in nursing duties among female healthcare workers; secondary analysis of a randomised controlled trial. BMC Musculoskelet Disord. 2019;20:328.

35. Backhausen MG, Bendix JM, Damm P, Tabor A, Hegaard HK. Low back pain intensity among childbearing women and associated predictors. A cohort study. Women Birth. 2019;32:e467-e476.

36. Robson EK, Kamper SJ, Davidson S, et al. Healthy Lifestyle Program (HeLP) for low back pain: protocol for a randomised controlled trial. BMJ Open. 2019;9(9):e029290.

37. Williams A, Lee $\mathrm{H}$, Kamper SJ, et al. Causal mechanisms of a healthy lifestyle intervention for patients with musculoskeletal pain who are overweight or obese. Clin Rehabil. 2019;33:1088-1097. 